\title{
Fission Time Measurements: A New Probe into Superheavy Element Stability
}

\author{
M. Morjean, ${ }^{1, *}$ D. Jacquet, ${ }^{2}$ J. L. Charvet, ${ }^{3}$ A. L'Hoir, ${ }^{4}$ M. Laget, ${ }^{2}$ M. Parlog,,${ }^{1,5}$ A. Chbihi, ${ }^{1}$ M. Chevallier, ${ }^{6}$ C. Cohen, ${ }^{4}$ \\ D. Dauvergne, ${ }^{6}$ R. Dayras, ${ }^{3}$ A. Drouart, ${ }^{3}$ C. Escano-Rodriguez, ${ }^{1}$ J. D. Frankland, ${ }^{1}$ R. Kirsch, ${ }^{6}$ P. Lautesse, ${ }^{6}$ L. Nalpas, ${ }^{3}$ \\ C. Ray, ${ }^{6}$ C. Schmitt, ${ }^{6}$ C. Stodel, ${ }^{1}$ L. Tassan-Got, ${ }^{2}$ E. Testa, ${ }^{6}$ and C. Volant ${ }^{3}$ \\ ${ }^{1}$ GANIL, CEA-DSM, and IN2P3-CNRS, B.P. 55027, F-14076 Caen Cedex, France \\ ${ }^{2} I P N O, C N R S / I N 2 P 3$, Université Paris-Sud 11, F-91406 Orsay Cedex, France \\ ${ }^{3}$ CEA-Saclay, IRFU/Service de Physique Nucléaire, F-91191 Gif sur Yvette Cedex, France \\ ${ }^{4}$ INSP, Université P. et M. Curie-Paris 6, UMR 7588 CNRS, 140 rue de Lourmel, F-75015 Paris, France \\ ${ }^{5}$ National Institute for Physics and Nuclear Engineering, RO-76900 Bucharest-Magurele, Romania \\ ${ }^{6} I P N L$, Université Lyon 1, CNRS/IN2P3, 4 rue E. Fermi, F-69622 Villeurbanne, France
}

(Received 26 February 2008; published 11 August 2008)

Reaction mechanism analyses performed with a $4 \pi$ detector for the systems ${ }^{208} \mathrm{~Pb}+\mathrm{Ge},{ }^{238} \mathrm{U}+\mathrm{Ni}$ and ${ }^{238} \mathrm{U}+\mathrm{Ge}$, combined with analyses of the associated reaction time distributions, provide us with evidence for nuclei with $Z=120$ and 124 living longer than $10^{-18} \mathrm{~s}$ and arising from highly excited compound nuclei. By contrast, the neutron deficient nuclei with $Z=114$ possibly formed in ${ }^{208} \mathrm{~Pb}+\mathrm{Ge}$ reactions have shorter lifetimes, close to or below the sensitivity limit of the experiment.

DOI: 10.1103/PhysRevLett.101.072701

The fusion reactions between two heavy nuclei to form superheavy elements (SHEs) with $Z \gtrsim 110$ are strongly hindered by competing faster processes. At bombarding energies well above the fusion barrier, the fission barriers vanish due to spin effects, leading to fast splitting of the composite systems [1] before full equilibration. At energies close to the fusion barrier, the so-called quasifission (QF) process, an out of equilibrium binary splitting of the composite system [2-4], becomes dominant. Since its discovery, QF has been the object of many experimental [5-10] and theoretical studies [11-15]. For very heavy systems, fragments arising either from QF, after full kinetic energy damping, or from fusion followed by fission (FuFis) have very similar properties. Therefore, any kinematical discrimination between highly probable QF events and possible FuFis events is impossible.

Actually, the essential difference between the two processes resides in the involved reaction time $\left(t_{\text {reac }}\right)$. QF is mostly dynamical, whereas in FuFis, the system is trapped behind a potential barrier, the fission barrier, and relies on thermally driven shape fluctuation to escape this barrier. Very short QF time scales ( $\lesssim 10^{-20} \mathrm{~s}$ ) have been quite directly determined from the highly anisotropic angular distributions of the emerging fragments [3,4]. FuFis reaction times result essentially from (i) the fission times $t_{\text {stat }}$ due to the finite lifetimes $\tau_{f}$ of the compound nucleus $(\mathrm{CN})$ and of its daughters and (ii) the deformation times between the saddle and scission points. Even though the most probable $t_{\text {stat }}$ can be rather short, the exponential radioactive decay law can give rise to tails [16], mainly arising from daughter nuclei, extending to times much longer than those characteristic of QF. The saddle to scission times might be comparable to the shortest $t_{\text {stat }}$ due to the rather long involved path for SHEs, but are negligible with respect to the longest $t_{\text {stat }}$, provided realistic dissipation is considered $[17,18]$. Recently, attempts have been made to
PACS numbers: 25.70.Jj, 24.75.+i, 25.70.Lm, 27.90.+b

discriminate between $\mathrm{QF}$ and FuFis from the prescission neutron multiplicities, considered as a chronometer $[9,10]$. In the present Letter, capture reactions (CAPT), in which all the projectile and target protons form a composite system that will dominantly split in two heavy fissionlike fragments, have been identified with a $4 \pi$ charged product detection array. Evidences for fusion have been searched for among CAPT reactions from the associated reaction time distributions, especially from tails at very long times measured by the blocking technique in single crystals.

Three systems have been studied at GANIL: ${ }^{208} \mathrm{~Pb}+\mathrm{Ge}$ at $6.16 \mathrm{MeV} / u,{ }^{238} \mathrm{U}+\mathrm{Ni}$ at $6.62 \mathrm{MeV} / u$, and ${ }^{238} \mathrm{U}+\mathrm{Ge}$ at $6.09 \mathrm{MeV} / u$, possibly leading to CNs with $Z_{\mathrm{CN}}=114$, 120 and 124, respectively. Reverse kinematics permit measurements of fission fragment atomic number $(Z)$ and kinetic energy $(E)$, allowing reaction mechanism analyses. The blocking technique permits a quite direct discrimination between reactions faster and longer than a limit $t_{\text {lim }}$ corresponding to the time needed by the composite systems to move away from the thermal vibration domain of the atoms of the crystal [19]. Reverse kinematics, associated to the detection angle, lead to $t_{\mathrm{lim}} \approx 10^{-18} \mathrm{~s}$ for the three systems, at least 2 orders of magnitude longer than the measured QF characteristic times. The chosen high bombarding energies increase by orders of magnitude the typical fusion cross sections involved in synthesis experiments $[20,21]$. However, due to the target thicknesses ( $2 \mu \mathrm{m}$ for the 3 targets), the $\mathrm{CN}$ excitation energy distributions are broad, extending up to $\sim 80 \mathrm{MeV}$. For such high excitation energies, the shell effects possibly stabilizing SHEs [20,21] should be partly washed out [22]. Consequently, the $Z_{\mathrm{CN}}$ values have been chosen in the region of $Z$ shell closure(s) predicted between $Z=114$ and $Z=126$, depending on the models [23-28]. Indeed, the residual shell effects at a given temperature should be at the highest in this region, thus maximizing the tails at long 
fission times. The experiment is therefore probing fission barriers which only exist due to shell effects.

The three systems have been studied with the same setup. To avoid radiation damage, the beam impact on the crystal was moved about every 2 hours. The beam intensity was maintained at $\sim 10^{7} \mathrm{pps}$. Two extra beams ( $\mathrm{Kr}$ and $\mathrm{Xe}$ ) impinging on $\mathrm{Ge}, \mathrm{Ni}, \mathrm{Au}, \mathrm{Ag}$, or $\mathrm{Cu}$ targets with different thicknesses were also used for $E$ and $Z$ calibrations. Three telescopes, each consisting of a low pressure $\left(\mathrm{CF}_{4}\right.$ at 30 torrs) ionization chamber followed by a $5 \times 5 \mathrm{~cm}^{2} 2$-dimensional position sensitive silicon detector, were located at about $1 \mathrm{~m}$ from the target. Their angular, energy, and charge resolutions were better than $0.02^{\circ}, 2 \%$, and \pm 2 charge units up to $Z=92$, respectively. Two telescopes were located at $20^{\circ}$ with respect to the beam axis, essentially for fissionlike fragment detection. The third one pointed to $11^{\circ}$ in order to control on-line the crystal quality with elastic scattering. For blocking measurements, a $\langle 100\rangle$ axis of the Ge crystals or a $\langle 110\rangle$ axis of the Ni crystal was oriented towards one of the telescopes at $20^{\circ}$. All coincident charged products were detected and identified by INDRA [29] in a solid angle close to $4 \pi$. We thus controlled the involved reaction mechanisms and could reject off-line any incomplete fusion reactions, sequential fission of projectilelike fragments ... that might be mistaken with compound nucleus fission.

A complete description of the blocking technique can be found in $[19,30]$. It requires a precise measurement of the angular distribution as a function of the angle $\psi$ between the directions of the crystal axis and of the detected fragment. The blocking effects give rise to a dip in this distribution with a minimum yield $\chi_{\min }$ precisely in the direction of the crystal axis and a width varying as $(Z / E)^{1 / 2}$. For perfect crystals and experimental conditions, $\chi_{\text {min }}$ reaches for $t_{\text {reac }}<t_{\text {lim }}$, its minimum possible value that does not depend, due to its geometrical origin (the thermal vibration amplitude), on the nature ( $Z$ and $E$ ) of the blocked ions. However, in practice, the dips are filled and broadened due to crystal defects or experimental conditions (beam size, determination of the axis direction...), leading thus to an increase of the measured $\chi_{\min }$ value with respect to the one expected for perfect conditions. This $\chi_{\min }$ increase will obviously be larger for narrower dips. Therefore, in the following, direct evidences for reaction times longer than $t_{\mathrm{lim}}$ in a given selection of events will be considered as reliable only if the associated $\chi_{\min }$ is significantly greater than a reference $\chi_{\min }$ obtained for $t_{\text {reac }} \ll$ $t_{\lim }$ and if this reference is taken from a dip with a similar or a narrower width. More quantitative information has been sought from simulations of the dips by a Monte Carlo procedure following each atomic interaction between fragments and the crystal atoms. Parameters for these simulations (beam spot size, bending and defects of the crystal, response function of the detectors) have been included together within a single instrumental response function determined by fitting dips measured for $t_{\text {reac }} \ll t_{\text {lim }}$. The sensitivity of the simulations to the ingredients of the calculations (effective atomic interaction, taken within Thomas Fermi approximation, screening effects, charge state distributions of the nascent fragments, charge states all along the path in the crystal, post-scission emission, time distributions assumed with exponential shapes....) has been carefully checked and cannot modify in a sensitive way our conclusions.

Figure 1 presents the $Z$ versus $E$ distributions measured at $20^{\circ}$ by the blocking telescope. For the two heaviest systems, deep-inelastic reactions $\left(\mathrm{DI}_{\mathrm{proj}}\right)$ can be identified around the projectile atomic numbers. Just below, bananashaped distributions correspond to heavy fissionlike fragments $\left(\mathrm{CAPT}_{\text {heavy }}\right)$ arising from CAPT (only two heavy fragments with $Z>2$ detected with kinematical characteristics expected for fission fragments). For $\mathrm{Pb}+\mathrm{Ge}$, the $\mathrm{DI}_{\text {proj }}$ and $\mathrm{CAPT}_{\text {heavy }}$ branches merge together below $550 \mathrm{MeV}$. A clean discrimination between the two mechanisms is thus achievable only for $E \gtrsim 550 \mathrm{MeV}$. For this system, the complementary fissionlike fragment $\mathrm{CAPT}_{\text {light }}$ is around $Z=40$ at high and low $E$. For the two heaviest systems, the $\mathrm{CAPT}_{\text {light }}$ region is highly dominated by sequential fission of uraniumlike fragments (SeqFis). Taking into account that INDRA angular coverage is not actually $100 \%$, a clean $\mathrm{CAPT}_{\text {light }}$ selection could not be achieved for these systems. At the target atomic numbers, quasielastic scattering $\left(\mathrm{QE}_{\text {targ }}\right)$ gives rise to intense peaks with tails of more inelastic reactions at lower $Z$, except for $\mathrm{Pb}+\mathrm{Ge}$ where the target is scattered at more backward angles. Depending on the system considered, different limits have been applied on $Z$ and $E$ to select reaction mechanisms.

Figure 2 presents blocking dips measured around $20^{\circ}$ for various reaction mechanisms. Quite different shapes are observed, arising partly from the different ratios $(Z / E)^{1 / 2}$ involved, from the crystal characteristics (for instance mosaic structure of the Ni crystal broadens the dips and makes their slopes softer), or from different radiation

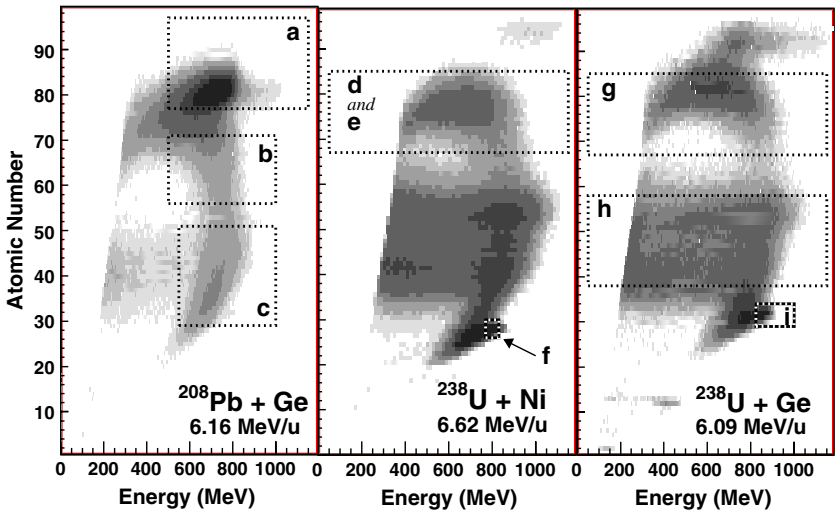

FIG. 1 (color online). Atomic number versus energy measured at $20^{\circ}$ (log scale) above the identification thresholds. The boxes labeled from (a) to (i) show the event selections for the dips of Fig. 2. 


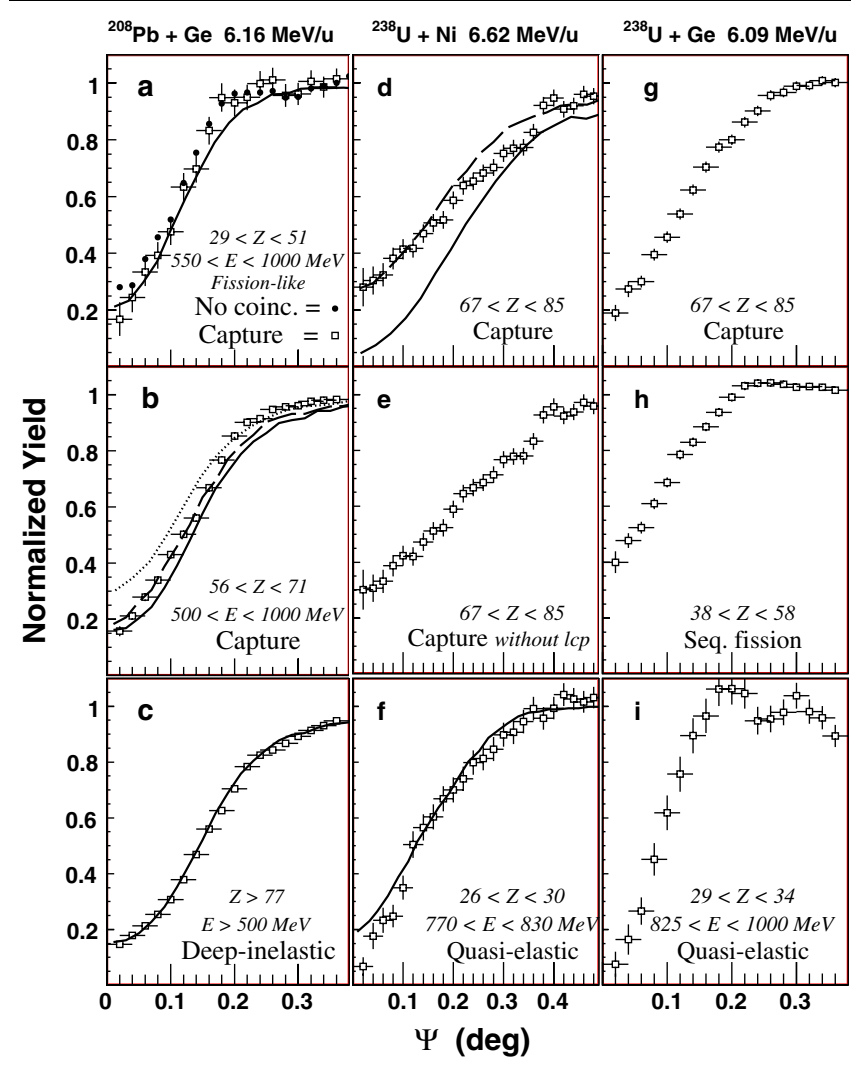

FIG. 2 (color online). Blocking dips at $20^{\circ}$. The horizontal bars indicate the angular range of integration. The full curves correspond to simulations for $t_{\text {reac }} \ll 10^{-18} \mathrm{~s}$ (see text for the other curves). The labels (a) to (i) refer to the event selections shown in Fig. 1.

damage suffered. However, particularly for $\mathrm{U}+\mathrm{Ge}$, the shapes are also modified by unforeseen charge collection defects around thin polarized aluminum strips implanted each $5 \mathrm{~mm}$ on the $1 \mu \mathrm{m}$ mylar windows of the blocking ionization chamber to render the transverse electric field more uniform. These defects lead to errors on $Z$ identification, up to 2 units for the highest $Z$. No satisfying corrections could be applied due to a complex dependence on the fragment charge and energy. Therefore, when $Z$ or $E$ limits are applied, nonstatistical fluctuations of the yields arise due to border effects at angles corresponding to position of the strips. They become large for narrow $Z$ or $E$ selections, when border effects are important. For the two lightest systems, due to crystal curvatures, the target translations made to limit the effect of radiation damage resulted in angular displacements of the crystal axis direction within the telescope acceptance. The effects of charge collection distortion averaged over a large area of the ionization chamber aperture resulted in negligible shape modifications of the blocking dips. By contrast, for $\mathrm{U}+$ $\mathrm{Ge}$, due to a weaker curvature, the crystal axis direction was only slightly modified for each beam impact. It intercepted the ionization chamber window just in between two strips, ensuring us that the central region of the dips is not affected and thus giving access to meaningful $\chi_{\min }$ estima- tions for the different reaction mechanisms considered. However, the region above the half width can be strongly affected, as it can be clearly seen for instance on the plateau in Fig. 2(i): the fluctuations can reach up to $10 \%$ of the yield, leading to an uncertainty larger than $30 \%$ on the dip width at half its minimum. Because of these large uncertainties on the shapes at large angles, no meaningful shape simulation could be undertaken for $\mathrm{U}+\mathrm{Ge}$.

For $\mathrm{Pb}+\mathrm{Ge}$, similar $\chi_{\min }$ values are found for $\mathrm{CAPT}_{\text {light }}, \mathrm{CAPT}_{\text {heavy }}$ and the very fast $\mathrm{DI}_{\text {proj }}$ reactions [open squares in Figs. 2(a)-2(c)], thus indicating no reaction times significantly longer than $t_{\min }$ for CAPT reactions. The full, dashed, and dotted curves in Fig. 2(b) correspond to simulations assuming lifetimes $\tau \ll t_{\text {lim }}$, $\tau=10^{-18} \mathrm{~s}$ and $\tau=2 \times 10^{-18} \mathrm{~s}$, respectively. The instrumental response function has been determined from the fit shown in Fig. 2(c) (full curve). The best fit in Fig. 2(b) is for $\tau=10^{-18} \mathrm{~s}$ whereas, for the complementary light fragment, it is obtained for $\tau<t_{\text {lim }}$ [full curve in Fig. 2(a)]. Such a behavior points for this system to $t_{\lim } \sim$ $10^{-18} \mathrm{~s}$, as expected, and to capture reaction times shorter than or close to this value. The high sensitivity of the technique to long lifetimes is demonstrated by the dip presented as full dots in Fig. 2(a): the same $Z$ and $E$ limits as for $\mathrm{CAPT}_{\text {light }}$ have been applied to select fissionlike events, but no coincidence with INDRA is now required to identify CAPT reactions. For clarity, the error bars, similar to those associated to the open squares, have been suppressed. Without coincidence, a significant dip filling is observed with respect to the $\mathrm{CAPT}_{\text {light }}$ dip for $\psi<0.1^{\circ}$ due to a very weak proportion of leadlike fission. Unfortunately, clean reaction mechanism selections are not always achieved in fission time experiments.

The two heavier systems have rather similar behaviors. References for $t_{\text {reac }} \ll t_{\text {lim }}$ can be obtained from $\mathrm{QE}_{\text {targ }}$ in Figs. 2(f) or 2(i) [for $\mathrm{U}+\mathrm{Ge}$, a similar $\chi_{\min }$ reference value is obtained from $\mathrm{DI}_{\text {proj }}$ despite the quite different $(Z / E)^{1 / 2}$ ratio, indicating for this target that no significant spurious broadening affects the measured $\chi_{\min }$. As shown by Fig. 2(h) for $U+\mathrm{Ge}$, the highly probable SeqFis reactions are associated with strong $\chi_{\min }$ increases with respect to the short time references, in agreement with [16]. Figures 2(d) and 2(g) show that, for both systems, sizeable $\chi_{\min }$ increases are also observed for $\mathrm{CAPT}_{\text {heavy }}$. They are far above any experimental or statistical uncertainties, whatever the possible extrapolations towards $\psi=0^{\circ}$. Figure 2(e) shows that removing from the analysis all the CAPT events with a coincident light charged particle does not affect the dips, despite sizable measured multiplicities ( 0.10 for $Z=120$ and 0.08 for $Z=124$ ). Simulations show that post-scission neutron emission cannot significantly modify the $\chi_{\min }$ value, as confirmed by selecting highly excited targetlike fragments that lead to the same $\chi_{\min }$ value as for the weakly excited fragments in $\mathrm{QE}_{\text {targ }}$. Therefore, the $\chi_{\min }$ measured for $\mathrm{CAPT}_{\text {heavy }}$ provide us with unique model independent evidences for nuclei with 
$Z=120$ and 124 that undergo fission at times much longer than $10^{-18} \mathrm{~s}$. Furthermore, considering more and more asymmetric splitting by increasing the lower $Z$ limit of the $\mathrm{CAPT}_{\text {heavy }}$ selection result in constant $\chi_{\text {min }}$ (or slightly increased within statistical errors), clearly pointing to asymmetric fission of $Z=120$ and 124 . Taking into account the statistical and systematic errors on the yields as well as errors arising from the extrapolation towards $\psi=$ 0 , the most pessimistic estimations lead to a minimum percentage of $(10 \pm 5) \%$ of nuclei living more than $10^{-18} \mathrm{~s}$ among the capture reactions for both $Z=120$ and 124.

For $\mathrm{U}+\mathrm{Ni}$, an instrumental response function has been determined by fitting the $\mathrm{QE}_{\text {targ }}$ dip with $\tau \ll t_{\text {lim }}$ [full curve in Fig. 2(f)]. Applying this instrumental function, simulations for $\mathrm{CAPT}_{\text {heavy }}$ assuming $\tau<t_{\text {lim }}$ [full curve in Fig. 2(d)] can definitively not reproduce the data, whereas a satisfactory fit is obtained assuming $\tau=2.2 \times 10^{-18} \mathrm{~s}$ (dashed curve). However, more complex reaction time distributions with different proportions of shorter and longer times also reproduce the measured dip, leading in any case to average reaction times longer than $\tau=2.2 \times$ $10^{-18} \mathrm{~s}$. The minimum yield simulated for $\mathrm{QE}_{\text {targ }}$ events is larger than the one for $\mathrm{CAPT}_{\text {heavy }}$ with $\tau \ll t_{\text {lim }}$ due to the broadening resulting from the strong mosaic structure of the nickel crystal. Considering now as the actual reference for $t_{\text {reac }}<t_{\text {lim }}$ the $\chi_{\text {min }}$ simulated for $\mathrm{CAPT}_{\text {heavy }}$, the minimum percentage of CNs living more than $10^{-18} \mathrm{~s}$ increases up to about $20 \%$. It must be stressed that the percentages and reaction times inferred from the present experiments are only valid at $20^{\circ}$ due to different $\mathrm{QF}$ and FuFis angular distributions.

Very long fission times can only show up if the fission barriers of all the isotopes involved in the decay chain are high enough to allow significant fast particle emission before fission. For $Z=114$, the small percentage of long fission time events (if any) might thus arise from low fission barriers due to neutron deficiency with respect to predicted neutron magic numbers $(N=180$ or 184) [2328]. The short measured time scale shows that the long reaction times cannot result only from dynamical evolutions as suggested in [31]. By contrast, the large proportions of long lifetime components for the much less neutron deficient $Z=120$ and 124 nuclei provide us with evidence for high fission barriers, not compatible for $Z=124$ with the very low shell energies calculated in [23], but in qualitative agreement with expected shell closures between $Z=120$ and 126 [26-28]. Despite kinematical limitations due to the backward detection angle that does not give access to the most asymmetric scissions, the fission observed for $Z=120$ and 124 is clearly asymmetric, suggesting a rapid cooling before scission that restores shell effects. This work shows that the tails at very long times in the scission time distributions are powerful probes into enhanced stability regions for superheavy elements. Studies as a function of the atomic number, the neutron number, and the temperature will bring unique information on the SHE stabilizing effects: islands of stability around magic spherical nuclei, large shell gaps due to deformation effects, isomeric effects, fission barrier temperature dependence.... Theoretical efforts are, however, clearly needed to reproduce both the fission time scales and the tiny residue cross sections.

We wish to thank the INDRA collaboration for giving us access to their $4 \pi$ detector. We specially thank M. F. Rivet for her help during the analysis of ICs detectors. Many thanks are also due to J.F. Berger and D. Boilley for fruitful discussions.

*morjean@ganil.fr

[1] W. U. Schröder and J. R. Huizenga, Treatise on Heavy-Ion Science (Plenum Press, New York, 1984), Vol. 2.

[2] R. Bock et al., Nucl. Phys. A 388, 334 (1982).

[3] J. Toke et al., Nucl. Phys. A 440, 327 (1985).

[4] W. Q. Shen et al., Phys. Rev. C 36, 115 (1987).

[5] J. Nestler et al., Phys. Rev. C 51, 2218 (1995).

[6] D. J. Hinde et al., Nucl. Phys. A 592, 271 (1995).

[7] B. B. Back et al., Phys. Rev. C 53, 1734 (1996).

[8] M. G. Itkis et al., Nucl. Phys. A 787, 150C (2007).

[9] T. Materna et al., Nucl. Phys. A 734, 184 (2004).

[10] R. Thomas et al., Phys. Rev. C 75, 024604 (2007).

[11] V. Zagrebaev and W. Greiner, J. Phys. G 31, 825 (2005).

[12] Y. Aritomo, Nucl. Phys. A 780, 222 (2006).

[13] A. Diaz-Torres, Phys. Rev. C 69, 021603 (2004).

[14] V. Y. Denisov and W. Nörenberg, Eur. Phys. J. A 15, 375 (2002).

[15] G. G. Adamian et al., Nucl. Phys. A 618, 176 (1997).

[16] F. Goldenbaum et al., Phys. Rev. Lett. 82, 5012 (1999).

[17] P. Fröbrich et al., Phys. Rep. 292, 131 (1998).

[18] Y. Abe et al., Phys. Rep. 275, 49 (1996).

[19] D. S. Gemmel, Rev. Mod. Phys. 46, 129 (1974).

[20] P. Armbruster, Annu. Rev. Nucl. Part. Sci. 50, 411 (2000).

[21] Y. Oganessian, J. Phys. G 34, R165 (2007).

[22] A. Ignatyuk, G. N. Smirenkin, and A. S. Tishin, Yad. Fiz. 21, 485 (1975).

[23] P. Møller et al., At. Data Nucl. Data Tables 59, 185 (1995).

[24] R. Smolanczuk, Phys. Rev. C 56, 812 (1997).

[25] S. Cwiok et al., Nucl. Phys. A 611, 211 (1996).

[26] M. Bender et al., Phys. Rev. C 60, 034304 (1999).

[27] J. F. Berger, D. Hirata, and M. Girod, Acta Phys. Pol. B 34, 1909 (2003).

[28] T. Bürvenich et al., Phys. Rev. C 69, 014307 (2004).

[29] J. Pouthas et al., Nucl. Instrum. Methods Phys. Res., Sect. A 357, 418 (1995).

[30] C. Cohen and D. Dauvergne, Nucl. Instrum. Methods Phys. Res., Sect. B 225, 40 (2004).

[31] J. U. Andersen et al., Phys. Rev. Lett. 99, 162502 (2007). 